\title{
Optimized Agglomeration Algorithmic rule to Uncover User Pattern Applying on Weblog Knowledge
}

\author{
Suman Lata Joshi \\ CSE Dept., GBPEC \\ Pauri, India
}

\author{
H S Bhadauria, Ph.D \\ CSE Dept., GBPEC \\ Pauri, India
}

\author{
Annapurna Singh, Ph.D \\ CSE Dept., GBPEC \\ Pauri, India
}

\begin{abstract}
These days, event logs contain immense amounts of knowledge that may simply overwhelm a personality's. Consequently, mining content from the phenomenon of logs is accepted as a crucial function for the system management. This paper presents a completely unique clump rule for $\log$ file information sets that helps one to sight frequent patterns from $\log$ files, to create $\log$ file profiles, and to spot abnormal $\log$ file lines. To attain this, clump tool is employed for playacting 2 phases. First, a military operation is finished on internet group action information. Second, AN analysis is finished on internet access log files to research the user group action behavior and user's log-in approach. During this paper, the cluster medical specialty and verification tool is employed to find hidden relationships among the net server information and access patterns.
\end{abstract}

\section{Keywords}

Cluster, Data Processing, Information Clump, Log file, System Observance, Tool, Web.

\section{INTRODUCTION}

In the field of System and network management Event work And log files are taking part in crucial role in system and network management. From the past twenty years, the BSD syslog etiquette [1] has grow to be a good accepted common place that is supported on varied operative systems Associate in Nursing is implemented in an extremely big scope of organization devices. Literate system applications either use the syslog protocol or end up log files in custom format, whereas many devices like routers, switches, optical device printers, etc. having the power to utilize the server host practice for the syslog etiquette.

Usually, content are logged as single-line substance messages. Since $\log$ files are an exquisite offer for crucial the health standing of the system, many sites have built a centralized work and $\log$ file observance infrastructure. Owing to the importance of $\log$ files as a result of the provision of system health information, style of tools are developed for observance of log files, e.g., Swatch [2], Log surfer [3], and SEC [4]. Log file observance techniques are also classified into fault detection and anomaly detection. Among the case of fault detection, the domain skilled creates a data of fault message patterns. The log file monitor takes a specific action whenever line is attached to a log file which is similar to a pattern.

This ordinarily used approach has one serious flaw - solely those faults that square measure already glorious to the domain professional are often detected. If an antecedently unknown fault condition happens, the log data monitor merely ignores the corresponding message within the log file, since there's no match for it within the pattern information. Also, it's typically tough to seek out an individual with decent data regarding the system. Within the case of anomaly detection, a system profile is made that reflects traditional system activity. If messages square measure logged that don't match the profile, associate alarm is raised. With this approach, antecedently unknown fault conditions square measure detected, however on the opposite hand, making the system profile by hand is long and erring.

\section{PROBLEM STATEMENT}

Firstly, quite many info sets embrace points with categorical attributes, where the domain of associate attribute could be a extent set of values $[13,14]$.As associate instance, take into account a right away info set with attributes car-producer, prototype, type, and color, and knowledge points ('Honda', 'Civic', 'hatchback', 'green') and ('Ford', 'Focus', 'sedan', 'red'). Also, it's quite common for categorical info that absolutely completely different points can have different type of attributes. Measuring the data between info points there are several normal distance functions for categorical info exist, such as a result of the Jaccard constant $[12,13])$, for chose the correct operator is often an uncommon task. Report it that the log data lines could also be as observe points from a categorical info set, since each line of data set could also be separated into words, where nth world define the usefulness for the nth attribute. There proposed square measure aiming to use this illustration of log file info among the rest of this paper.

Secondly, quite much info sets of late are high dimensional, where info points can merely have tens of attributes. Sadly, ancient clump methods square measure found to not work well once they are applied to high dimensional info. as a result of the variability of dimensions $\mathrm{n}$ can increase, it has always the case that for every mix of points there exist dimensions where these points are such a lot except each other, that creates the detection of any clusters nearly out of the question (according to some sources, this draw back starts to be severe once $n \geq 15)[12,15]$. What's a lot of, ancient clump methods are sometimes unable to sight natural clusters that exist in subspaces of the primary high-dimensional space [15]. As an instance, there are some info points $(1330,1,1,99,25,2033$, 1040), (12, 1, 1, 724, 668, 36, 2305), and (501, 1, 1, 1822, $1749,808,9867)$ are not present as a cluster by many ancient methods, since among the initial info they are not adjacent to each other. On the alternative hand, they have a positively dense cluster among the second and dimension of the realm.

The properties problems delineate beyond are relevant to the clump of $\log$ file info, since log file info is typically highdimensional and many of the road patterns correlated with clusters in different spaces 
For instance, the lines data connecting from 192.168.1.0 after that the Data give the result as RSA key prompting and Data secret authentication completed.

In the initial phase of paper there proposed an algorithm which is explained in section III. Experimental results are organized in section IV. At last there mention the future Scope of proposed algorithm.

\section{PROPOSED ALGORITHM}

The nature of the information in any clustered data set performs a key role when selecting the proper rule for clump. Many clustering operations outline for generic information sets like market basket information, wherever no specific assumptions regarding the character of knowledge are created. However, after that there examine the content of typical log files at the word level, there are necessary properties that distinguish log file information from a generic information set. Throughout this experiments there used six log file information sets from varied domains: horsepower Open read event log file, mail server log file, Squid cache server log file, net banking server log file, file and print server log file, and Win2000 domain controller log file.

Although it's not possible to verify that the properties got discovered here characterize each log file created on earth, the $\log$ file is common to a large vary of log file information sets. Firstly, majority of the words occur solely a couple of times within the information set. The results of a research for estimating the currency times of words in log file information. The results show that a majority of words were terribly sporadic, and a big fraction of words appeared one time within the information set (one may argue that almost all of the words occurring once are timestamps, however once timestamps were far from information sets, there determined no vital variations within the experiment results). Also, solely a little fraction of words were comparatively frequent, i.e., they occurred a minimum of once per each ten, 000 or 1,000 lines. Similar phenomena are determined for net information, wherever throughout a research nearly five hundredth of the words was found to occur once only [2].

Secondly, there discovered several robust correlations between words which occurred often. As this result is caused by the actual fact that a message is usually formatted in keeping with an explicit format string before it's logged, e.g., printf (message, "Connection occasionally port \%d", ip address, port number); once events of constant kind are logged over and over, constant components of the format string can become frequent words that take place over and over within the information set. Within the next subdivision there proposed clump rule that depends on the special properties of log file information.

\section{EXPERIMENT AND RESULT}

Our aim was to style a rule which might be quick and build solely a couple of passes over the info, and which might sight clusters that are gift in subspaces of the first information area. The rule depends on the special properties of log file information mentioned within the previous subdivision, and uses the density based mostly approach for clump. The information area is assumed to contain data points with categorical attributes, wherever every purpose represents a line from log file information set.

The element of information is the words from the corresponding log file line. The info area has $\mathrm{n}$ dimensions, wherever $\mathrm{n}$ is that the most variety of words per line within the information set. a part $\mathrm{S}$ may be a set of the info area, wherever bound attributes $\mathrm{i} 1, \ldots, \mathrm{ik}(1 \leq \mathrm{k} \leq \mathrm{n})$ of all points that belong to $\mathrm{S}$ have identical values $\mathrm{v} 1, \ldots, \mathrm{vk}: \forall \mathrm{x} \in \mathrm{S}$, xi1 $=\mathrm{v} 1$, ..., xik $=$ vk. There show the set of mounted attributes of region $\mathrm{S}$. If $\mathrm{k}=1$ (i.e., there's only 1 mounted attribute), the region is named 1-region. A dense region may be a region that contains a minimum of $\mathrm{N}$ points, wherever $\mathrm{N}$ is that the support threshold worth given by the user.

The rule consists of 3 steps just like the succulent rule [14] it 1 st makes a hop over the info and builds an information outline, and so makes another pass to create cluster candidates, mistreatment the outline info collected before. As a final step, clusters are chosen from the set of candidates. Throughout the primary step of the rule (data summarization), the rule identifies all smoggy 1-regions. Note that this task is reminiscent of the mining of frequent words from the info set (the word position within the line is taken under consideration throughout the mining).

A word is taken into account frequent if it happen minimum of $\mathrm{N}$ times within the information set, wherever $\mathrm{N}$ is that the user-specified support threshold worth. When smoggy 1regions (frequent words) are known, the rule made all cluster clients throughout one pass. The cluster clients are unbroken within the candidate table that is at the start empty. The data set is deal with it line by line, and if a line is be located at 1 or a lot of smoggy1-regions, a cluster candidate is created. If the cluster candidate isn't gift within the candidate table, it'll be inserted into the table with the support worth one, otherwise its support worth are going to be incremented. In each condition, the road is devoted to the cluster client. The cluster client is created within the following way:

If the road belongs to $\mathrm{m}$ smoggy 1-regions that have mounted attributes $(\mathrm{i} 1, \mathrm{v} 1), \ldots,(\mathrm{im}, \mathrm{vm})$, then the cluster clients might be a region with the set of mounted attributes. As an example, if the road is affiliation from 192.168.1.0, and there exist a smoggy 1-region with the mounted attribute (1, 'Connection') and another smoggy 1-region with the mounted attribute (2, 'from'), then a part with the set of mounted attributes becomes the cluster candidate. Standard priority rule for mining frequent item sets [18], since frequent words may be survey as frequent 1 -itemsets. Then, however, our rule takes a rather totally different approach, generating all cluster candidates quickly.

Table 1. Run Time Resemblance of Proposed Rule and a Prime-Concern Algorithmic Rule

\begin{tabular}{|l|l|l|l|l|l|}
\hline & $\begin{array}{l}\text { Under } \\
\text { pin } \\
\text { thresh } \\
\text { old } \\
\mathbf{5 0 \%}\end{array}$ & $\begin{array}{l}\text { Under } \\
\text { pin } \\
\text { thresh } \\
\text { old } \\
\mathbf{2 5 \%}\end{array}$ & $\begin{array}{l}\text { Under } \\
\text { pin } \\
\text { thresh } \\
\text { old } \\
\mathbf{1 0 \%}\end{array}$ & $\begin{array}{l}\text { Under } \\
\text { pin } \\
\text { thresh } \\
\text { old 5\% }\end{array}$ & $\begin{array}{l}\text { Under } \\
\text { pin } \\
\text { thresh } \\
\text { old 1\% }\end{array}$ \\
\hline $\begin{array}{l}\text { Propos } \\
\text { ed } \\
\text { Rule } \\
\text { for A }\end{array}$ & $1 \mathrm{sec}$ & $1 \mathrm{sec}$ & $1 \mathrm{sec}$ & $2 \mathrm{sec}$ & $2 \mathrm{sec}$ \\
\hline $\begin{array}{l}\text { Prime } \\
\text { concer } \\
\text { n for A }\end{array}$ & $2 \mathrm{sec}$ & $16 \mathrm{sec}$ & $96 \mathrm{sec}$ & $145 \mathrm{sec}$ & $\begin{array}{l}5650 \\
\mathrm{sec}\end{array}$ \\
\hline $\begin{array}{l}\text { Propos } \\
\text { ed } \\
\text { Rule } \\
\text { for B }\end{array}$ & $5 \mathrm{sec}$ & $5 \mathrm{sec}$ & $5 \mathrm{sec}$ & $6 \mathrm{sec}$ & $6 \mathrm{sec}$ \\
\hline $\begin{array}{l}\text { Prime } \\
\text { Conce } \\
\text { rn for }\end{array}$ & $9 \mathrm{sec}$ & $28 \mathrm{sec}$ & $\begin{array}{l}115 \\
\mathrm{sec}\end{array}$ & $206 \mathrm{sec}$ & $\begin{array}{l}2770 \\
\mathrm{sec}\end{array}$ \\
\hline
\end{tabular}




\begin{tabular}{|l|l|l|l|l|l|}
\hline B & & & & & \\
\hline $\begin{array}{l}\text { Propos } \\
\text { ed } \\
\begin{array}{l}\text { Rule } \\
\text { for C }\end{array}\end{array}$ & $10 \mathrm{sec}$ & $10 \mathrm{sec}$ & $12 \mathrm{sec}$ & $12 \mathrm{sec}$ & $13 \mathrm{sec}$ \\
\hline $\begin{array}{l}\text { Prime } \\
\text { Conce } \\
\text { rn for } \\
\text { C }\end{array}$ & $182 \mathrm{sec}$ & $182 \mathrm{sec}$ & $\begin{array}{l}18950 \\
\mathrm{sec}\end{array}$ & $\begin{array}{l}29062 \\
\mathrm{sec}\end{array}$ & $\begin{array}{l}427791 \\
\mathrm{sec}\end{array}$ \\
\hline
\end{tabular}

There are many reasons for that. Firstly, A priori rule is in terms of runtime $[7,8]$, since the candidate generation and testing involves exponential quality. Secondly, one effect of $\log$ data file information is that there are many vigorous collection between different words, it makes very little sense to check a doubtless immense variety of frequent word combos that are generated by A priori, whereas solely a comparatively little variety of combos are gift within the information set. It's rather cheaper to spot the present combos throughout one hop over the info, and verify when the pass that of them corresponds to clusters.

It ought to be noted that since A priori uses level-wise candidate generation, it's ready to sight patterns that our rule doesn't report. E.g., if words A, B, C, and D are frequent, and also the solely combos of them within the information set are A B C and A B D, then our rule won't examine the pattern A B. On the opposite hand, by proscribing the search our rule avoids news all subsets of a frequent items that may simply overwhelm the user, however rather aims at police work highest occurring item sets solely (several pattern-mining algorithms like Max-Miner [12] use the similar approach).

In order to match the runtimes of rule and a priori-based rule, there enforce each algorithm in Perl and tested them against 3 little $\log$ file information sets. Table two presents the results of tests that were conducted on one, $5 \mathrm{GHz}$ Pentium4 digital computer have $256 \mathrm{MB}$ of memory and Red hat eight.0 UNIX as OS (the sizes of $\log$ files $\mathrm{A}, \mathrm{B}$, and $\mathrm{C}$ were $183 \mathrm{~KB}$, $1812 \mathrm{~KB}$, and $4004 \mathrm{~KB})$.

The results obtained show that the clump rule is superior to the priori-based clump theme in terms of runtime price. The results conjointly indicate that a priori-based clump schemes are applicable just for little log file information sets and high support thresholds. Though proposed rule makes simply 2 passes over the info and is thus quick, it may consume plenty of memory once applied to a bigger information set. Within the next subdivision discuss the memory price problems in additional detail.

\section{CONCLUSION}

In the whole research Cluster medical specialty and Verification Tool was utilized. Within the analysis of the complete information set, there propose a rough plan of non member's reach quantitative relation for this website. The Cluster medical specialty and Verification tool capture log files moreover as a analyze log files that found to be quite helpful for the study of diary analysis.

\section{FUTURE SCOPE}

Since during their proposed cluster keeping with the chance distribution, in future work on rising the within cluster variation by 1 st sorting the objects in keeping with correlation inside every and so finding probability and running the scans with the higher than or any clump rule it will improve the standard of clusters and entropy of data gain from every cluster [7]

\section{REFERENCE}

[1] R. Agrawal and R. Srikant, pp.487. Fast algorithms for mining association rules.

[2] Jiawei Han, Micheline Kamber, Jian Pei, Data Mining: Concepts and Techniques.

[3] Vijay Laxmi2 and M. Afshar Alam3, Optimizing the web mining techniques using heuristic approach.

[4] Preeti Chopra, Md. Ataullah, Feb2013. A Survey on Improving the Efficiency of Different Web Structure Mining Algorithms. (IJEAT).

[5] Mr.Ramesh, Prajapati, April- 2012. A Survey Paper on Hyperlink Induced Topic Search (HITS) Algorithms for Web Mining. (IJERT)

[6] Darshna Navadiya, Roshni Patel, December-2012. Web Content Mining Techniques-A Comprehensive Survey. (IJERT).

[7] S. Kamruzzaman, Farhana Haider, Ahmed Ryadh Hasan, Text Classification Using Data Mining. ICTM.

[8] Ajit Abhraham, Vitorino Ramos, Web Usage Mining Using Artificial Ant Colony Clustering and Linear Genetic.

[9] Dec.2003. Programming, to appear in CEC'03 Congress on Evolutionary Computation. IEEE Press, Canberra, Australia, 8-12.

[10] Cyrus Shahabi, Amir M. Zarkesh, Jafar Adibi, and Vishal Shah, 1997. Knowledge Discovery from Users Web-page Navigation. IEEE RIDE.

[11] Margaret H. Dunham, MHD2003. Data Mining Introductory and Advanced Topics, Prentice Hall.

[12] Moe Moe Zaw, Ei Ei Mon, "Improved Cuckoo Search Clustering Algorithm (ICSCA)", Proceedings of the 11th International Conference on Computer Applications.

[13] X.-S. Yang, 2010. Nature-Inspired Met heuristic Algorithms. Max-Miner Press.

[14] F. Liu, C. Yu, and W. Meng, Jan.2004. Personalized Web Search for Improving Retrieval Effectiveness, IEEE Trans. Knowledge and Data Eng. vol. 16, no. 1, pp. 2840.

[15] T. Joachims, 2002. Optimizing Engines Using Click through Data. Proc. ACM SIGKDD.

[16] Koyoro Shadeo, June 2012. Trends in web Based Search Engine 'Journal of emerging trends in computing and information Sciences' .Vol. 3, No-6, ISSN - 2079-8407. 